\title{
BRIGITTA HAFTKA
}

\section{„Deutsch ist eine V/2-Sprache mit Verbendstellung und freier Wortfolge."}

\author{
Abstract \\ Der Titel des Beitrags vereinigt häufige typologische Einordnungen des Deut- \\ schen, die, wenn sie nicht interpretiert werden, einander widersprechen: \\ 1. Deutsch ist eine $S$ (ubjekt)O(bjekt)V(erb)-Sprache/eine OV-Sprache. \\ 2. Deutsch ist eine der germanischen Verbzweitsprachen. \\ 3. Deutsch ist eine Sprache mit freier Wortstellung. \\ 4. Deutsch ist eine Scramblingsprache.
}

Im ersten Teil gehe ich zunāchst auf die beiden Einordungen, die die Verbposition betreffen, ein und zeige, daB die dem eingeleiteten Nebensatz entsprechende SOV- oder OV-Reihenfolge bei Annahme eines nach links regierenden Verbs im Deutschen sinnvollerweise als grundlegend angesehen werden kann, während die den germanischen Verbzweitsprachen eigene Anordnung des finiten Verbs nach einer maximalen Konstituente als abgeleitet betrachtet werden muß. Im zweiten Teil wird gezeigt, wie die sich aus der Vergabe der semantischen Rollen (Thetarollen) des Verbs nach links ergebende hierarchische Struktur auf die lineare Folge im Mittelfeld deutscher Sätze abgebildet wird und wie man sich Umordnungen dieser Reihenfolge (oft als Scrambling bezeichnet) bei Berücksichtigung von Skopusverhältnissen (z.B. der Negation/Affirmation) sowie von Kontextbedingungen und speziellen Topikalisierungen vorstellen kann und wieso sie eingeschränkt - also nicht völlig frei - sind.

\section{1. (S)OV-Sprache vs. V/2-Sprache}

Wenn beide Kennzeichen gleichzeitig gelten sollen, kann das nur folgendermaßen verstanden werden: Wir wissen, daß das finite Verb im Deutschen, speziell im deklarativen Hauptsatz normalerweise immer einer maximalen Phrase (XP) folgt. Dies kann sowohl das Subjekt sein:

(1) [DPManche Linguisten] lieben Nonsens.

als auch jede andere maximale Phrase:

(2) [DPNonsens] hat Vater schon immer geliebt.

(3) [PPSeit seiner Kindheit [PP in den Bergen des Harzes]] wandert er gern.

einschließlich von verbalen Projektionen und Sätzen: 
(4) [VP-f;zuIm Harx zu wandern] macht Spaß.

(5) [VP-f Mannheim besuchen] wollte er schon lange.

(6) [ $\mathrm{CP}$ Daß wir unsere Kollegen hier wiedersehen], ist ein besonderes Vergnügen.

Diese Tatsache wird als Test dafür verwendet, ob eine sprachliche Sequenz eine maximale Phrase ist oder nicht. Was vor dem finiten Verb stehen kann, ist eine maximale Phrase:

(7) a. [AdvP Auf] steigt der Strahl und fallend gieft er voll der Marmorschale Rund ... (C.F. Meyer, Der Römische Brunnen)

b. [AP [DPSeiner Mutter] ähnlich] war Peter schon immer.

Auch im uneingeleiteten abhängigen Satz steht das finite Verb an zweiter Stelle:

(8) Er meinte, [DP Nonsens] habe Vater schon immer geliebt.

Das gilt auch für $w$-Phrasen, die in $w$-Interrogativsätzen ebenfalls dem finiten Verb vorangehen:

(9) [AdvP Wo] tagen heute die Linguisten?

(10) [PP Von wem] wurde die Tagung eröffnet?

(11) [DP Wessen Vorlesung] hat euch gefallen?

Im Gegensatz zu diesen Verbzweit-Sätzen steht das finite Verb in den eingeleiteten abhängigen Sätzen (12-19) an letzter Stelle:

Er meinte,

(12) daß manche Linguisten Nonsens lieben.

(13) daß Vater schon immer Berge geliebt hat.

(14) daß er seit seiner Kindheit Nonsens geliebt haben soll.

Ich weiß nicht,

(15) ob er schon lange Mannheim besuchen wollte.

(16) a. ob der Strahl aufsteigt.

b. ob Peter schon immer seiner Mutter ähnlich war.

(17) wo heute die Linguisten tagen.

(18) von wem die Tagung eröffnet wurde.

(19) wessen Vorlesung euch gefallen hat.

Warum wird nun davon ausgegangen, daß das Deutsche eine SOV- oder OV-Sprache sei, wenn doch diese Endposition des finiten Verbs meist nur in abhängigen Sätzen vorkommt? Dazu betrachten wir die Struktur der Verbalphrase im Satz (20): 


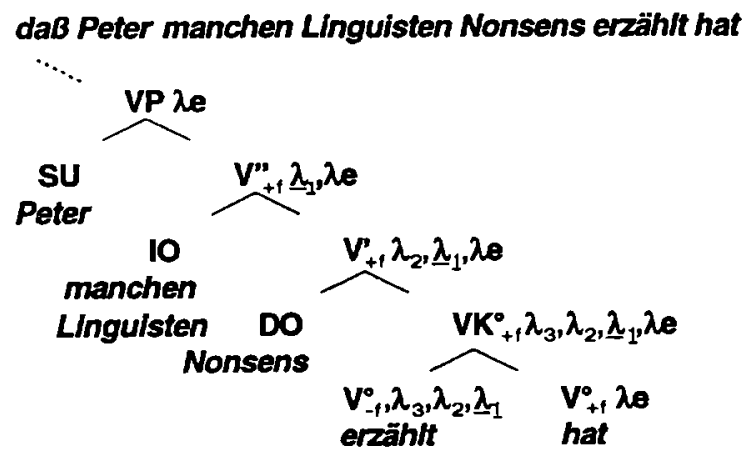

(S)

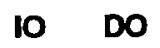

Wie (20) zeigt, steht das finite Auxiliar $\left[\mathrm{V}^{*}+\mathrm{f}\right.$ hat $]$ im Verbendsatz innerhalb seiner Verbalphrase, genauer innerhalb seines Verbkomplexes $\left[V^{\circ}+f\right.$ erzählt hat]. Der Verbkomplex (VK $\left.{ }^{\circ}\right)$ „erbt” - grob gesagt - neben den formalen Eigenschaften auch die referentielle Argumentstelle $\lambda$ e vom finiten Auxiliar und die Subkategorisierungseigenschaften, semantischen Rollen oder Theta-Rollen $\left(\lambda_{3}, \lambda_{2}, \lambda_{1}\right)$ vom Partizip Perfekt des Hauptverbs. Dies ergibt - nach Bierwisch (1990) - die Argumentstruktur des Verbkomplexes.

Die interne Theta-Rolle $\lambda_{3}$ wird vom $\mathrm{VK}^{\circ}$ an seine unmittelbar vorangehende Nachbarkonstituente, die DP Nonsens, vergeben. Mit der Vergabe dieser Thetarolle $\lambda_{3}$ an die als Thema fungierende DP ist der strukturelle Akkusativ verbunden. Das heißt, die DP fungiert als direktes Objekt (DO). Nimmt man nun an, daß der Verbkomplex durch die Vergabe dieser Thetarolle zu V' projiziert, so erhält man eine erste Projektionsstufe des Verbs, die wiederum die Theta-Rolle $\lambda_{2}$ an die als Adressat fungierende DP manchen Linguisten vergibt. Diese von V' vergebene interne Thetarolle, die hier mit dem Dativ verbunden ist, ist das indirekte Objekt (IO).

In manchen generativen Überlegungen ist damit die Verbalphrase abgeschlossen. Deshalb bezeichnet man die verbliebene mit dem Nominativ verbundene Thetarolle für das Agens $\lambda_{1}$ im Gegensatz zu den internen Thetarollen für die Objekte als externe Thetarolle. In neueren Phrasenstrukturmodellen wird häufig die externe Thetarolle an die im Spezifizierer der VP stehende SU-DP vergeben. Durch die Vergabe der externen 
Thetarolle projiziert V' (oder, je nach dem, wie man sich die VP-Struktur bei mehr als einem Objekt vorstellt, $V^{\prime 1}$ ) zur maximalen Verbalphrase VP.

VP hat nun noch die referentielle Argumentstelle, die, wie Ilse Zimmermann zeigt, „durch einen Operator zu spezifizieren ist, der den Referenztyp des betreffenden Syntagmas festlegt" (Zimmermann 1991, S. 4). Dies geschieht jedoch erst in einer späteren Stufe der Ableitung des Satzes.

Wenn das Verb nun im deutschen Nebensatz seine Thetarollen in der beschriebenen Weise nach links vergibt (s.(21)),

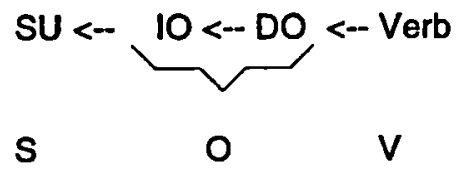

so ist es sinnvoll, anzunehmen, daß das Verb im Deutschen nach links $\Theta$-regiert, daß also den verschiedenen Reihenfolgen im deutschen Satz eine Reihenfolge SOV innerhalb der VP zugrundeliegt. ${ }^{2}$

Die normale Aussagesatz-Verbstellung $\mathrm{V} / 2$ ist dann als Abwandlung dieser Grundreihenfolge zu beschreiben, d.h. man muß eine Verschiebung des finiten Verbs aus seiner die hierarchische Struktur spiegelnden Endposition in eine Position nach der ersten maximalen Phrase und, wie die einleitenden Beispiele zeigen, nicht einfach nach dem Subjekt annehmen.

In der generativen Grammatik wird in verschiedenen Variationen angenommen, daB das finite Verb als Head der Verbalphrase (VP) seine morphologischen Merkmale im Head einer die VP dominierenden funktionalen Kategorie INFL (I) bzw. der Unterkategorien von INFL: Subjekt-

1 Auf die VP-Schalen von Larson (1988) soll hier nicht eingegangen werden.

2 Im Rahmen der generativen Grammatik wird zur Zeit heftig darüber debattiert, wie eine universelle Phrasenstruktur aussehen sollte. Vorausgesetzt, daß der Kopf einer Phrase ein Komplement und einen Spezifizierer haben kann,

- so wird nach der hier favorisierten Auffassung angenommen, daß deren Anordnung sprachspezifisch parametrisiert ist. Für das Deutsche gilt dann eine weitere Differenzierung nach sog. verbalen oder nominalen Kategorien:

+V-Kategorien (Verb, Adjektiv) $\Theta$-regieren ihre Komplemente nach links

$+\mathrm{N}$-Kategorien (Substantiv, Präposition) $\Theta$-regieren ihre Komplemente nach rechts 
und Objekt-Kongruenz (Agreement), Tempus (Tense) usw. abholen oder neuerdings (vgl. Chomsky 1992) spätestens in der LF-Komponente überprüfen oder checken muB. Das bedeutet, daß das finite Verb sukzessive an die jeweiligen Headpositionen adjungiert wird und auf diese Weise in eine Position links von der VP gelangt, nämlich nach $I^{\circ}$ (= AGRS). Beschränkt wird diese Bewegung durch den sog. HeadMovement-Constraint, der besagt, daB Heads - also $\mathrm{X}^{\circ}$-Kategorien - nur in eine $\mathrm{Y}^{\circ}$-Position verschoben werden dürfen, d.h. in die Head-Position einer hierarchisch höheren Kategorie. Betrachten wir die INFL-Phrase der Einfachheit halber hier als Blackbox, berücksichtigen wir also hier nicht die einzelnen Unterkategorien, so kann man sich diese Verbbewegung vorstellen wie in (22):

a.

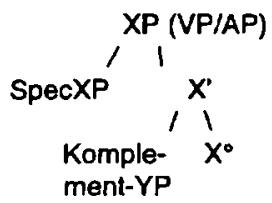

b.

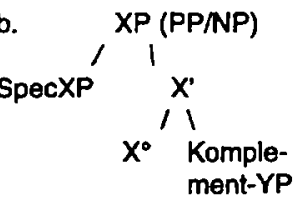

- Nach der anderen Auffassung (Kayne u. a.) gibt es nur eine universelle Phrasenstruktur, in der der Spezifizierer stets links und das Komplement stets rechts vom Kopf steht.

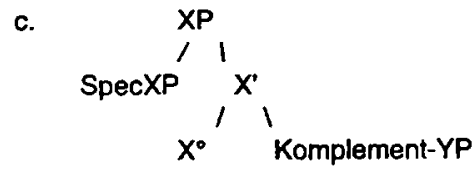

Im ersteren Falle ist die $\Theta$-Rektionsrichtung des Kopfes innerhalb einer angenommenen VP-Struktur parametrisiert und damit typologisch relevant. Im zweiten Falle wäre die Rektionsrichtung des Verbs typologisch irrelevant. Die Struktur der VP wäre in allen Sprachen gleich. Die typologische Einordnung des Deutschen müßte von Oberflācheneigenschaften der Sätze abgelesen werden.

Siehe zu den Auswirkungen einer solchen universellen Phrasenstrukturannahme für die Beschreibung des Deutschen z.B. auch die Diskussion in Donati/Tomaselli (1995) und in C.M. Schmidt (1995). 


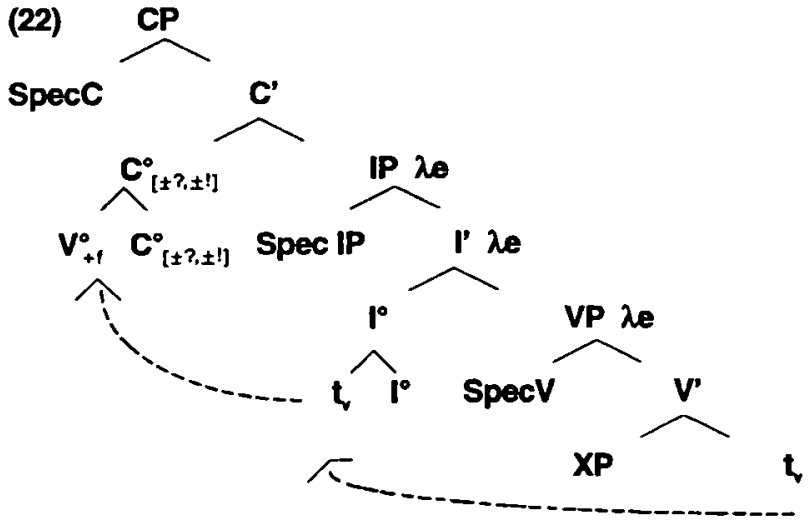

Nimmt man unter simplifizierender Vernachlässigung der Differenzhypothese $^{3}$ an, daß der Satz immer eine COMP-Phrase (CP) ist und daB in $C^{*}$ der an die Position des finiten Verbs gekoppelte Referenztyp des Satzes (Deklarativsatz, Interrogativsatz usw.) codiert ist, so wird die oben schon genannte referentielle Argumentstelle durch den Operator in $\mathrm{C}^{\circ}$ gebunden. Im Komplementsatz ist dies der Komplementierer daß oder $o b$.

Ist in $\mathrm{C}^{\circ}$ ein Satztyp codiert (z.B. durch $\left.[+?,-!]\right)$, der die oberflächensichtbare Anordnung des finiten Verbs in der zweiten Position versus in der ersten Position erfordert, so muß das Verb sich aus seiner $\mathrm{V}^{\circ}$ Position über die INFL-Head-Positionen in die höchste Head-Position, also in das lexikalisch leere $\mathrm{C}^{\bullet}$ bewegen und an dieses adjungieren. Dadurch wäre - stark vereinfacht - die Satztypcharakteristik z.B. eines Entscheidungsinterrogativsatzes gesichert:

(23) a. $\left[\mathrm{CP} / \mathrm{C}^{\prime}\left[\mathrm{C}^{*}\right.\right.$ Lieben $\left[\mathrm{C}^{*} \mathrm{I}_{+}\right.$?;-!] 0$] \mathrm{IP}_{\mathrm{IP}}\left[\mathrm{I}^{\prime} t_{\mathrm{v}} / \mathrm{VP}\right.$ manche Linguisten $[\mathrm{v}$ ' Nonsens $t[\mathrm{v}] J]]]]]]$ ?

3 Die hybride Differenzhypothese (2. Alternative), wie sie von Brandt et al. (1992) vertreten wird, besagt, daB

- Verb-letzt-Deklarativsātze und Verb-letzt-w-Interrogativsätze CP/IPs,

- Verb-zweit-Deklarativsätze und Verb-zweit-w-Interrogativsätze IPs,

- Verb-letzt-Entscheidungsinterrogativsātze $C^{1} / I^{1}$,

- Verb-erst-Deklarativsätze und Verb-erst-Entscheidungsinterrogativsātze $\mathrm{I}^{1}$ sind. 
Für V/2-Sätze muß mindestens und höchstens eine Phrase aus VP in die Spezifiziererposition der CP, also in eine Position vor das finite Verb gebracht werden.

Gibt es eine $w$-Phrase im Satz und ist $\mathrm{C}^{\bullet}$ als interrogativ [+?,-!] ausgezeichnet, so verlangt die Spezifizierer-Head-Kongruenz, daB diese $w$ Phrase in die Spezifizierer-Position der COMP-Phrase wandert (24):

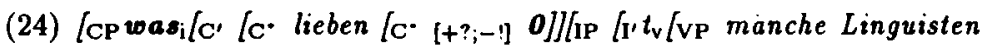
$\left.\left.\left[\mathrm{v}, t_{\mathrm{i}} t_{\mathrm{v}} J\right] J\right] J\right]$ ?

Ist $C^{*}$ dagegen als deklarativ $[-?,-!]$ ausgezeichnet, so verlangt die Spezifizierer-Head-Kongruenz, daB entweder eine - $w$-Phrase, d.h. eine maximale Phrase, die kein Fragewort enthält, nach Spec-CP verschoben wird, siehe (25) mit der Baumstruktur (26) und die Beispiele (1-8):

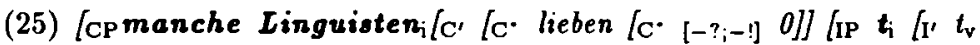
[vP $t_{i}\left[v^{\prime}\right.$ Nonsens $\left.\left.\left.\left.\left.t_{v}\right] J\right]\right]\right]\right]$
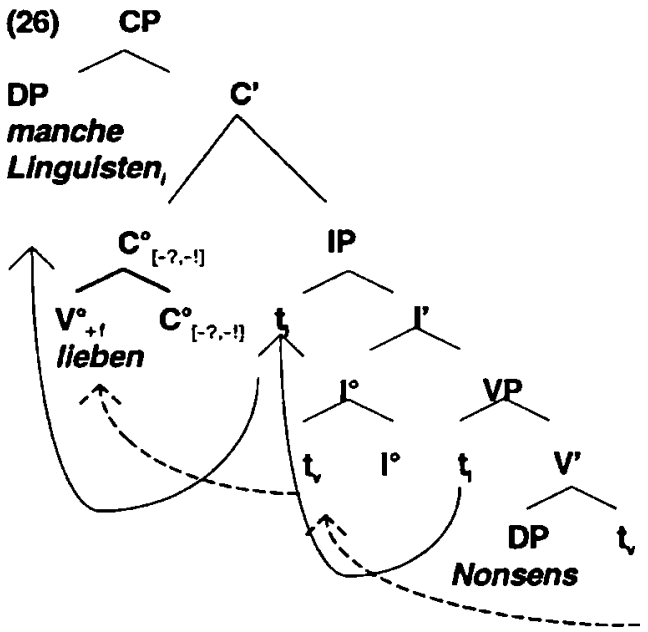

oder daß in SpecCP wie in (27) ein expletives ES eingesetzt wird, s.u.:

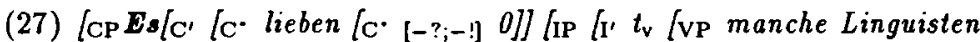
$\left[\mathrm{v}\right.$ Nonsens $t_{\mathrm{v}}$ [J]J]]

Diese Linksverschiebungen des finiten Verbs nach $\mathrm{C}^{\circ}$ und der maximalen Phrase nach SpecCP finden natürlich nur unter der Voraussetzung 
statt, daß $\mathrm{C}^{\bullet}$ zwar Satzmodus-Merkmale enthält, die aber lexikalisch unausgedrückt ( 0 ) bleiben. Das ist auch der Fall bei den uneingeleiteten abhängigen Sätzen, wie z.B. in unserem obigen Beispiel (8):

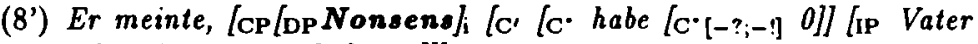
schon immer $t_{\mathrm{i}}$ geliebt $\left.t_{\mathrm{v}} \mathrm{Jl}\right]$

Steht in $\mathrm{C}^{\bullet}$ ein Komplementierer, also eine Konjunktion daß oder $o b$ wie in unseren Beispielen (12) - (16), so charakterisiert der Komplementierer den folgenden Satz als deklarativ oder interrogativ, und die Spezifiziererposition bleibt in der Standardsprache obligatorisch unbesetzt:

(12') [ $\mathrm{CP} / \mathrm{C}^{\prime}\left[\mathrm{C}^{\cdot}[-? ;-!]\right.$ daß] [IP manche Linguisten Nonsens lieben]]]

Ich gehe hier nicht auf die zahlreichen Besonderheiten ein, die mit der Besetzung der $\mathrm{C}^{\circ}$ - und der SpecCP-Position verbunden sind. Mehr darüber s. Haftka (1993) sowie Brandt/Reis/Rosengren/Zimmermann (BRRZ) (1992), die eine wichtige Alternative zu dieser hier nur angedeuteten traditionelleren Auffassung bieten (Differenzhypothese, s. Anm. 3)).

Man sieht, daB bei Annahme von projizierenden funktionalen Kategorien oberhalb der Verbalphrase, deren Head- und Spezifizierer-Merkmale die Veränderung der Verbposition und die Linksverschiebung einer XP veranlassen, relativ gut zu erklären ist, welches Verhältnis zwischen SOV und V/2-Stellung besteht, daB es sich dabei also um zwei verschiedene Ebenen handelt:

- (S)OV betrifft die Basisanordnung in der VP. Man findet dafür in der typologischen Literatur (vgl. Hawkins 1983, S. 230) auch Kleinschreibung: (s)ov, die als Hinweis auf die dargestellte Problematik zu deuten ist.

- $V / 2$ und auch $V / 1$ sind in einem solchen generativen Modell Derivationsergebnisse. Die Besetzung des lexikalisch leeren $\mathrm{C}^{*}$ durch das finite Verb und die angedeuteten Spezifizierer-HeadKongruenz-Eigenschaften müssen sprachspezifisch parametrisiert sein. Das heiBt, die Verschiebung des Verbs in die V/2- oder V/1Position hat, da sie ja in der Oberflächenstruktur des Satzes sichtbar ist, vor der Eingabe der derivierten Struktur in die Phonologische Komponente der Grammatik zu erfolgen. 


\section{Freie Wortstellung vs. „Scrambling”-Sprache}

Zwei weitere typologische Einordnungen des Deutschen lauten:

3. Deutsch ist eine Sprache mit freier Wortstellung.

4. Deutsch ist eine Scramblingsprache.

Satz 3, der behauptet, Deutsch habe eine freie Wortstellung, und Satz 4, der Deutsch als eine Sprache mit „durcheinandergebrachter” (= gescrambelter) Anordnung der Konstituenten charakterisiert, scheinen sich im Ergebnis zunächst nicht zu widersprechen, nur daß Satz 4 möglicherweise eine geordnete Grundreihenfolge voraussetzt, während Satz 3 nicht unbedingt eine solche Voraussetzung macht. Dies allerdings ist wichtig für die Deskription der deutschen Wortstellung. Die Abfolge von Subjekt, Objekt(en) und Adverbialen ist zwar nicht so streng geregelt wie im Englischen ${ }^{4}$, diese "Freiheit” ist aber durchaus strengen Regeln unterworfen.

Um das zu zeigen, gehen wir zurück zu unserer Struktur der VP: Mit der Vergabe der Argumentstellen des Verbs an die Komplemente und an das Subjekt ist ein Gerüst für den Satz, genauer für die Verbalphrase entstanden, das auf den verschiedenen Stufen durch adverbiale Modifikatoren erweitert werden kann. Dies geschieht normalerweise durch Adjunktion. Der Einfachheit halber nehme ich hier an, dab Modifikatoren an die verschiedenen V-Projektionsstufen basis-adjungiert werden können, in (28) also an V' die modale Adverbialbestimmung [Pp mit viel Humor] und an VP z.B. das Frequenzadverbiale [AdvP ofi]. Eine Anmerkung: Von den Modifikatoren zu unterscheiden ist z.B. die direktive adverbiale PP [PP vor die Nase]: deren direktive Präposition vor wird vom Verbkomplex [gesetzt hat] subkategorisiert, und sie vergibt ihrerseits ihre interne Thetarolle (Direktivum) an die DP [DPdie Nase]. Die PP ist also ein Komplement des Verbs, da ihr Head P vom Verb subkategorisiert ist, sie hat aber selbst keinen Argumentstatus.

4 Das Deutsche kann auf die Kennzeichnung der syntaktischen Funktion der Argumentphrasen als Subjekt (SU), indirektes Objekt (IO) oder direktes Objekt (DO) durch feste Positionen weitgehend verzichten, weil es eine ausreichende Substantiv- und vor allem Artikelflexion hat, die es ermöglicht, den Kasus einer DP auch in derivierter Position zu identifizieren. Bei Eigennamen ohne Artikel muß jedoch oft eine SU-IO-DO-Reihenfolge eingehalten werden, um Mißverstāndnissen vorzubeugen, vgl. Dann hat Hans Franz Maria vorgestellt. 


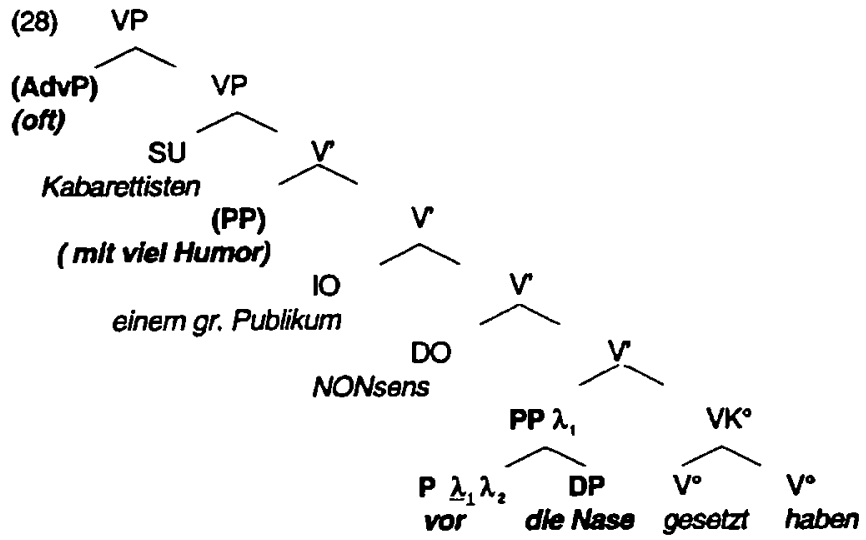

Wir haben also nun einen Sachverhalt, dergestalt daß

(29) oft Kabarettisten mit viel Humor einem großen Publikum Nonsens vor die Nase gesetzt haben.

Damit ist noch nichts darüber gesagt, ob dieser Sachverhalt bejaht oder verneint werden soll, ob er also im Skopus eines Negations- oder Affirmationsoperators stehen, ihm also folgen muß. In syntaktischen Termen ausgedrückt, bedeutet das, daß der Negations- oder Affirmationsoperator die VP c-kommandiert. ${ }^{5}$ Der Affirmationsoperator ist entweder phonetisch stumm oder wird durch eine Partikel (wie z.B. wohl, aber auch kaum) bzw. eine Partikelkombination (wie $2 . B$. $\left[\left[\mathrm{Pt}^{\cdot} \cdot \operatorname{sehr}\left[\mathrm{Pt}^{\cdot} \cdot\right.\right.\right.$ wohl $]$ ) lexikalisiert. Der Negationsoperator ist entweder ebenfalls durch eine Partikel wie nicht ausgedrückt, oder er ist in eine nie-oder kein-Phrase (keiner, niemand) integriert. Nimmt man an, daß der die negierende oder affirmierende Position zum Sachverhalt angebende Operator, wenn er leer ist oder durch eine Partikel lexikalisiert ist, als eine minimale Kategorie $\left(\mathrm{X}^{\circ}\right.$-Kategorie) zu einer maximalen Phrase projiziert, so ergibt sich die Positionsphrase (30).

5 x c-kommandiert nach Chomsky (1986) y, gdw.:

i. $x$ y nicht dominiert und

ii. wenn jedes $\mathrm{z}$, das $\mathrm{x}$ dominiert, auch $\mathrm{y}$ dominiert. 


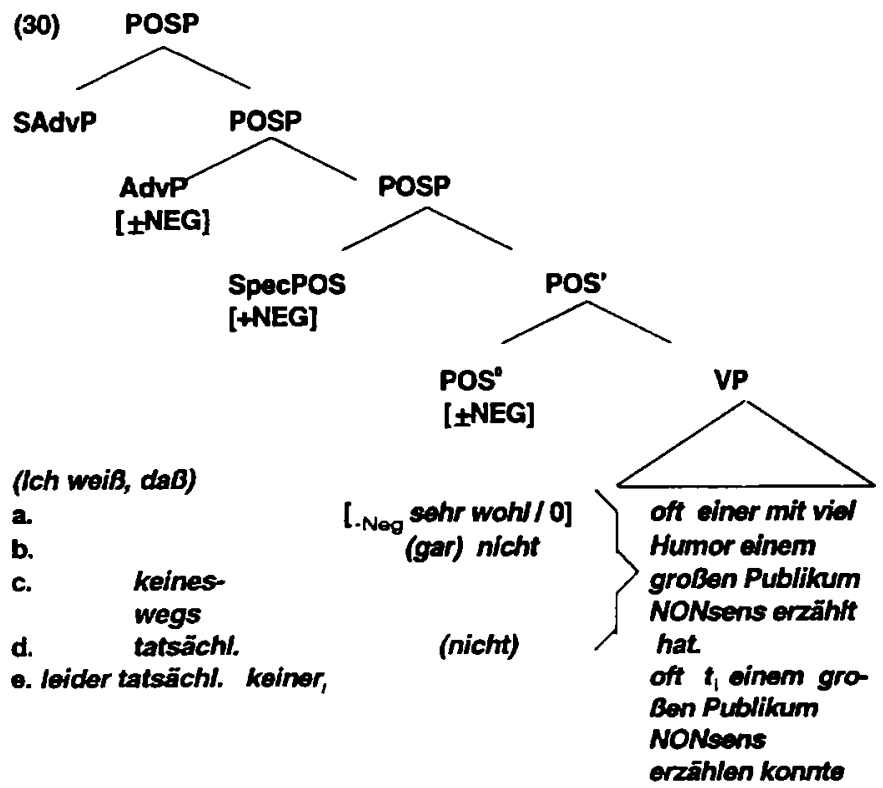

Ist die negierte Phrase eines der Elemente aus VP, so muß sie, soll die gesamte Proposition negiert sein, die VP verlassen, damit ihr [+NEG]Merkmal den Rest der VP in ihren Skopus nehmen kann, vgl. das keiner in (30e), das in die Spezifiziererposition der funktionalen Kategorie POSP oberhalb der VP wandern muß, vgl. die unterschiedlichen Skopusverhältnisse in (30e) und (30e'):

(30) e. daß leider tatsächlich keiner $r_{\mathrm{i}}\left[\mathrm{vp}\right.$ oft $t_{\mathrm{i}}$ einem großen Publikum NONsens erzählt hat]

'daß tatsächlich für keinen gilt, daß er oft einem großen Publikum Nonsens erzählt hat'

e'. daß leider tatsächlich [vpoft keiner einem großen Publikum Nonsens erzählen konnte]

'daß tatsächlich gilt, daß oft keiner einem großen Publikum Nonsens erzählen konnte'

Die die Negation oder Affirmation der Proposition modifizierenden Positionsadverbialia (tatsächlich, keinesfalls, keineswegs ...) werden an die Positionsphrase basis-adjungiert (30c-e). 
Nimmt man nun eine VP-Struktur wie (29) - ergänzt durch eine POSP wie (30) - an, so ergibt sich eine direkt der hierarchischen Struktur entsprechende Reihenfolge, die ich als Grundreihenfolge bezeichne. In solch einer Positionsphrase wird der Hauptakzent des Satzes dem syntaktisch zu bestimmenden Fokusexponenten zugewiesen, in (30) der am tiefsten eingebetteten subkategorisierten lexikalischen Nachbarkonstituente des Verbkomplexes ${ }^{6}$, nämlich NONsens.

Dieselbe Reihenfolge wird auch von definiten Substantiven eingehalten, die Bekanntes, aber nicht unmittelbar vorher schon einmal Erwähntes bezeichnen, also zum Fokusbereich gehören:

(31) (Ich habe gehört,)

daß (gestern die Chefin des Hauses charmant dem Publikum den jungen AUtor vorstellte]

Weitere wichtige Veränderungen dieser Grundreihenfolge - neben der Linksverschiebung einer negierten Phrase nach SpecPOS - ergeben sich bei der Anpassung eines Satzes an den Diskurs. Diese Anpassung ist im Deutschen und Niederländischen mit Linksverschiebungen von in der VP generierten Konstituenten innerhalb des Mittelfeldes verbunden, die man häufig alle oder nur teilweise als Scrambling bezeichnet, wobei Scrambling meist mit Adjunktion gleichgesetzt wird. Ich will hier nicht auf (mehr oder weniger kontrastive) Umordnungen mit lediglich informationsstrukturellen Konsequenzen eingehen, die nach Rosengren innerhalb der VP stattfinden und nach ihrer Meinung die einzig legitimen Scramblingfälle sind. In einem Syntaxmodell, das nach Chomsky (1992) nur Checking formaler Merkmale als Motiv für Bewegung zuläBt, habe ich dafür in Haftka (1994) eine Kontrastfokusphrase ${ }^{7}$ angesetzt, die der POSP übergeordnet ist und eine Spezifiziererposition für eine zwischen dem Satzadverbiale und der Negation/Affirmation anzuordnende sekundär betonte maximale Phrase zur Verfügung stellt:

(32) daß /FOCcP natürlich /FOCcP einem seRIÖsen Publikum / FOCc $^{\prime}$

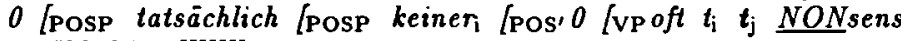
erzählt hätte]J]J]J]

6 Auf die Festlegung des grammatisch zu definierenden Fokusexponenten im Satz kann hier nicht eingegangen werden. $\mathrm{Vgl}$. dazu unter vielen anderen B. Haftka in: Grundzüge einer deutschen Grammatik (1981, S. 741-751), W. Abraham (1992), Rosengren (1994).

7 Die Kontrastfokusphrase ist an die Annahme gebunden, daB neben morphologischen Merkmalen (wie AGR(eement), T(ense)) auch das frei zugewiesene Kontrastfokus-Merkmal in einer entspechenden Spezifiziererposition gecheckt werden $k a n n / m u f$. 
So entsteht die für solche Sätze typische Hutkontur (Kapitälchen (sekundärer) - unterstrichene Großbuchstaben (primärer Akzent)).

An die Kontrastfokusphrase wird das Satzadverbiale basis-adjungiert, das auf diese Weise die gesamte affirmierte oder negierte Proposition subjektiv bewertend in seinen Skopus nimmt, s. (30e). Ist kein Kontrastfokusmerkmal und damit auch keine Kontrastfokusphrase im Satz vorhanden, so kann man annehmen, dab das Satzadverbiale an POSP adjungiert wird, worauf wir uns hier der Übersichtlichkeit halber beschränken wollen.

Ein anderer Komplex von Linksverschiebungen aus der VP betriftt diejenigen maximalen Phrasen, die auf Individuen referieren, die im unmittelbar vorangehenden Kontext oder in der Gesprächssituation schon "gegeben" sind, und deshalb als für den Hörer bewuBtseinspräsent und in diesem hörerbezogenen (Molnár (1991)) Sinne als „thematisch" angesehen werden. Solche Umordnungen können dazu führen, daB die POSP, vom Verb und von den Adverbialen abgesehen, teilweise oder völlig lexikalisch entleert wird. Die verschobenen Konstituenten hinterlassen an ihrer ursprünglichen Position koindizierte Spuren $\left(t_{i}, t_{j}\right)$ :

(33) Ich habe gehört,

a. daß die Chefin den Leuten $n_{\mathrm{j}}$ [angeblich [gestern $t_{\mathrm{i}}$ charmant $t_{\mathrm{j}}$ den jungen AUtor vorstellte]]

b. daß die Chefin diesen Mann $n_{\mathrm{i}}$ [angeblich [gestern $t_{\mathrm{i}}$ charmant dem PUBlikum $t_{\mathrm{k}}$ vorstellte]]

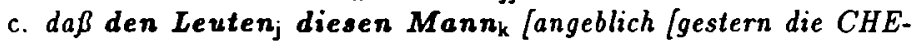
fin des Hauses $\mathrm{t}_{\mathrm{j}} \mathrm{t}_{\mathrm{k}}$ vorstellte]]

d. daß die Chefin $n_{\mathrm{i}}$ den Leuten diesen Mann $_{\mathrm{k}}$ [angeblich [gestern $\mathrm{t}_{\mathrm{i}} \mathrm{t}_{\mathrm{j}} \mathrm{t}_{\mathrm{k}}$ charmant VORstellte]]

Auch unbetonte Personal- oder Demonstrativpronomen müssen über das Satzadverbiale "gehoben" werden, also links vom Satzadverbiale stehen, vgl. $(33 e, f)$ :

(33) e. daß sie ihnen den $_{\mathrm{k}}$ [angeblich [gestern $t_{\mathrm{i}}$ charmant $t_{\mathrm{j}} t_{\mathrm{k}}$ VORstellte]]

f. daß ihnen den $n_{\mathrm{k}}$ langeblich [gestern die Chefin des Hauses charmant $t_{\mathrm{j}} t_{\mathrm{k}}$ VORstellte]]

gegenüber dem ungrammatischen (33e'):

(33) $\mathrm{e}^{\prime} .{ }^{*} \mathrm{da \beta}$ [angeblich [gestern sie charmant ihnen den vorstellte]]

Die Syntaxdeskription muB nun folgender Tatsache Rechnung tragen: Die Reihenfolge der unbetonten Konstituenten links vom Satzadver- 
biale/links von Modalpartikeln wie $j a / d o c h$ ist nicht beliebig, sondern wortform- und kasusabhängig, das heißt, es gibt eine Präzedenzhierarchie für gescrambelte Phrasen, links vom Satzadverbiale, die - sehr grob gesagt, - darauf hinausläuft, daß sich hier ebenfalls die Reihenfolge SU - IO - DO ergibt, auBer wenn es sich um Personalpronomen handelt, für die SU - DO - IO gilt.

Auf die Einzelheiten, wie diese Zusammenhänge beschrieben werden können, will ich hier nicht eingehen. Zwei Ansätze aber will ich nennen:

- Die der Vorstellung vom Deutschen als einer Scramblingsprache am nächsten kommende Alternative wäre eine Adjunktion an IP. Bei dieser Annahme kommt man nicht umhin, Filter zu formulieren, die sichern, daB die genannte Präzedenzhierarchie eingehalten wird ( $\mathrm{vgl}$. dazu das Filtersystem in Haftka 1988a, b).

- Die andere Alternative wäre, daB (wie in (34)) die verschiedenen Unterkategorien von INFL (SU-Kongruenz, IO-Kongruenz, DOKongruenz, Tempus), die als funktionale Kategorien zu maximalen Phrasen projizieren, Spezifizierer-Positionen für die oberflächensichtbare Kasusüberprüfung zur Verfügung stellen. ${ }^{8}$ Das würde bedeuten, daß Kasusüberprüfung nur für solche DPs oberflächensichtbar ist, die vor einem (möglichen) Satzadverbiale und damit vor der Positionsphrase und vor an die VP adjungierten Adverbialen stehen müssen, weil sie auf Vorerwähntes referieren und nicht im Skopus der Negation/Affirmation erscheinen dürfen, wenn sie nicht kontrastiv interpretiert werden sollen (Haftka 1994, 1995).

8 Die Annahme der Kongruenz- oder Agreement-Phrasen geschieht hier in Anlehnung an die Checking-Theorie des Minimalistischen Programms von Chomsky (1992), wobei es allerdings kaum empirische Evidenz dafür gibt, daß es im Deutschen neben dem overten Agreement-Checking bei diesen unbetonten vorerwähnten Phrasen auch noch ein verdecktes Checking der Kasusmerkmale aller anderen Argument-DPs auf dem Wege zur LF gibt. Das heißt, hier wird - Haftka $(1994,1995)$ wieder aufnehmend - nur versucht, die angeblich universellen Agreement-Spezifizierer für die Beschreibung der Reihenfolgerestriktionen gescrambelter Phrasen zu nutzen und zu zeigen, wo die Grenzen dieses Verfahrens liegen. 


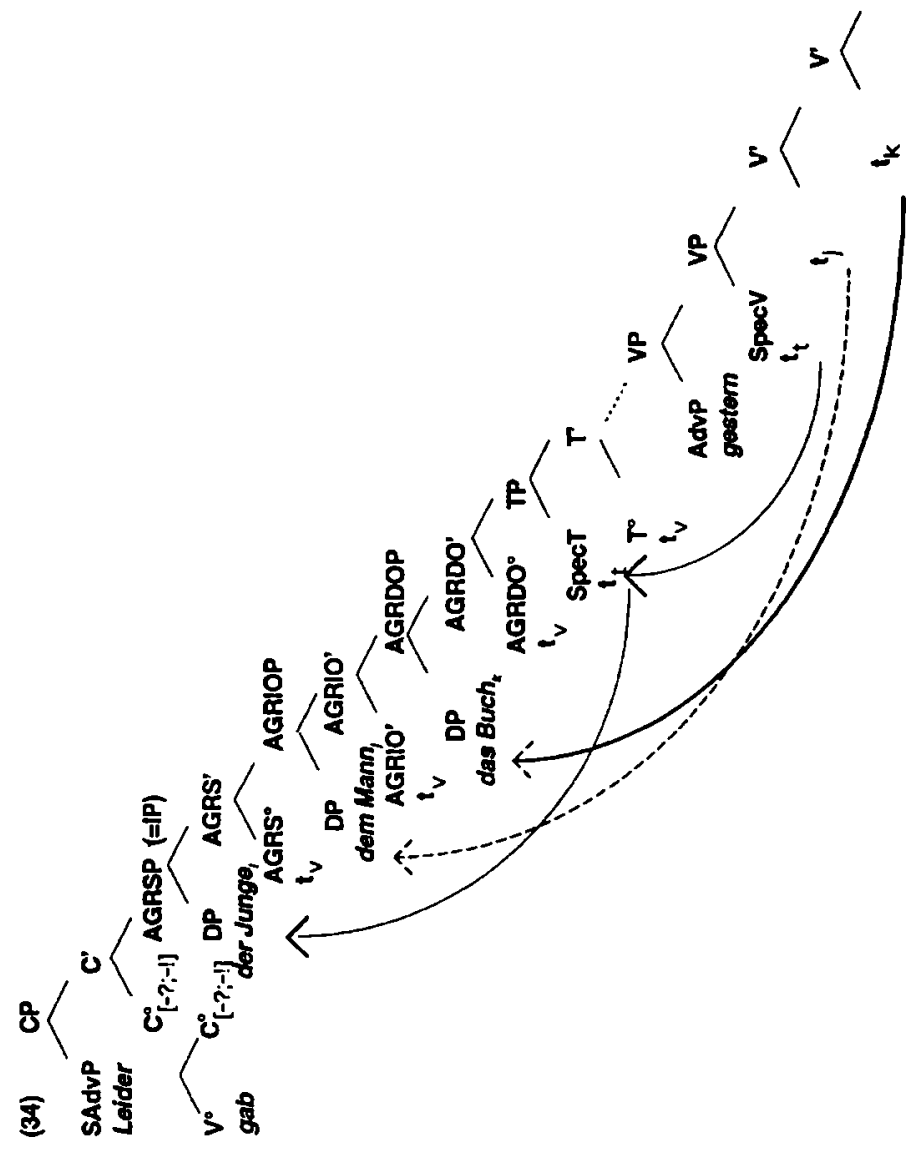


Rechnet man das durch, so bleibt auch dabei ein Problem offen, nämlich die idiosynkratisch feste Reihenfolge: akkusativisches Personalpronomen vor dativischem (35):

(35) a. daß $d u_{\mathrm{i}} i h n_{\mathrm{k}} i h m_{\mathrm{j}} \mathrm{t}_{\mathrm{i}}$ leider gestern $\mathrm{t}_{\mathrm{i}} \mathrm{t}_{\mathrm{j}} \mathrm{t}_{\mathrm{k}}$ GABst

b. daß $d u_{\mathrm{i}}$ dem $_{\mathrm{j}}$ das $_{\mathrm{k}} \mathrm{t}_{\mathrm{i}}$ leider gestern $\mathrm{t}_{\mathrm{i}} \mathrm{t}_{\mathrm{j}} \mathrm{t}_{\mathrm{k}}$ GABst

gegenüber der normalen Reihenfolge Dativ vor Akkusativ im Falle der Demonstrativpronomen (35b) und der definiten Nomina (34). Nimmt man einen Checking-Mechanismus wie in (34) an, so müBte das indirekte Objekt immer vor dem direkten Objekt erscheinen. Möglicherweise ist die DO-IO-Anordnung der Objekte ebenso wie die Klitisierung z.B. an $d a \beta$ in $(36 \mathrm{a})$ oder an $d u$ in $(36 \mathrm{~b})$

(36) a. dasses ihm sicher einer gegeben hat

b. daß du's ihm sicher gegeben hast

jedoch ein phonologischer ProzeB, der durch Prinzipien der PFKomponente abgedeckt wäre, also keinen Einwand gegen die Checkingtheorie darstellen müßte. ${ }^{9}$

Ich verwende hier für diese Linksverschiebungen im Mittelfeld nicht den Begriff „Scrambling”, weil es sich in dem angenommenen Modell nicht um Adjunktion etwa an IP handelt.

Nach meinen Beobachtungen (seit Haftka 1988) gibt es noch eine weitere Möglichkeit, die Grundreihenfolge SOV innerhalb der Verbalphrase zu verändern. Unter anderem kann dies auch dadurch geschehen, daB eine der Phrasen als Topik ausgezeichnet ist, d.h. als Gegenstand, über den bzw. als Situation, über die etwas ausgesagt werden soll. Das ist zwar häufig das Subjekt des Satzes ${ }^{10}$, kann aber auch eines der Objekte oder, wie ich annehme, auch eine der auf eine Situation referierenden adverbialen Phrasen (Temporale, Kausale ...) sein, unabhängig davon, ob das

$9 \mathrm{Vgl}$. auch H. Wegener (1985). In der Diskussion nach diesem Vortrag wies W. Abraham mich auf seinen interessanten Erklārungsansatz in Abraham (1995, S. 517) hin, der davon ausgeht, daB unbetonte Personalpronomina klitischen Charakter haben: „Der strukturelle Akkusativ steht dem CLTräger, vermittelt durch das funktionale Kongruenzmerkmal (AGR-O), nāher als der lexikalische Dativ". Eine Annahme, die aber bezogen auf (34) weitere Zusatzannahmen erfordert.

10 Statistiken, die ganze Texte untersuchen, kommen meist auf ca. $60 \%$ aller Sätze, die mit dem Subjekt beginnen. Ich kann solche Angaben aus einer eigenen unveröffentlichten statistischen Untersuchung aus den 60er Jahren (Novellen und Zeitungstexte aus der Goethezeit und der Gegenwart) bestätigen. 
Bezeichnete kontextuell oder situativ bewußtseinspräsent ist oder nicht. Eine solche Phrase, die einen Gegenstand/eine Situation bezeichnet, über den/die etwas ausgesagt werden soll und die deshalb das Merkmal [+Topik] erhält ( = „sachbezogene Ebene” bei Molnár 1991) kann zwischen $d a \beta$ oder dem Verb einerseits und den vorerwähnten Phrasen links vom Satzadverbiale andererseits erscheinen.

Wenn in einem syntaktischen Modell Verschiebungen von maximalen Phrasen immer nur dadurch zustande kommen, daB ein Spezifizierermerkmal einer funktionalen Kategorie das gleiche Merkmal hat wie die jeweilige Phrase, dann folgt aus der Tatsache, daB in einer Sprache eine Phrase mit einem bestimmten Merkmal wie z.B. Topik immer eine bestimmte Position einnimmt, daB dort die Spezifiziererposition sein muB, in der dieses Merkmal überprüft werden muB. Das heißt, zwischen $\mathrm{C}^{\circ}$ und IP/ AGRSP müssen wir eine Topikphrase annehmen. 


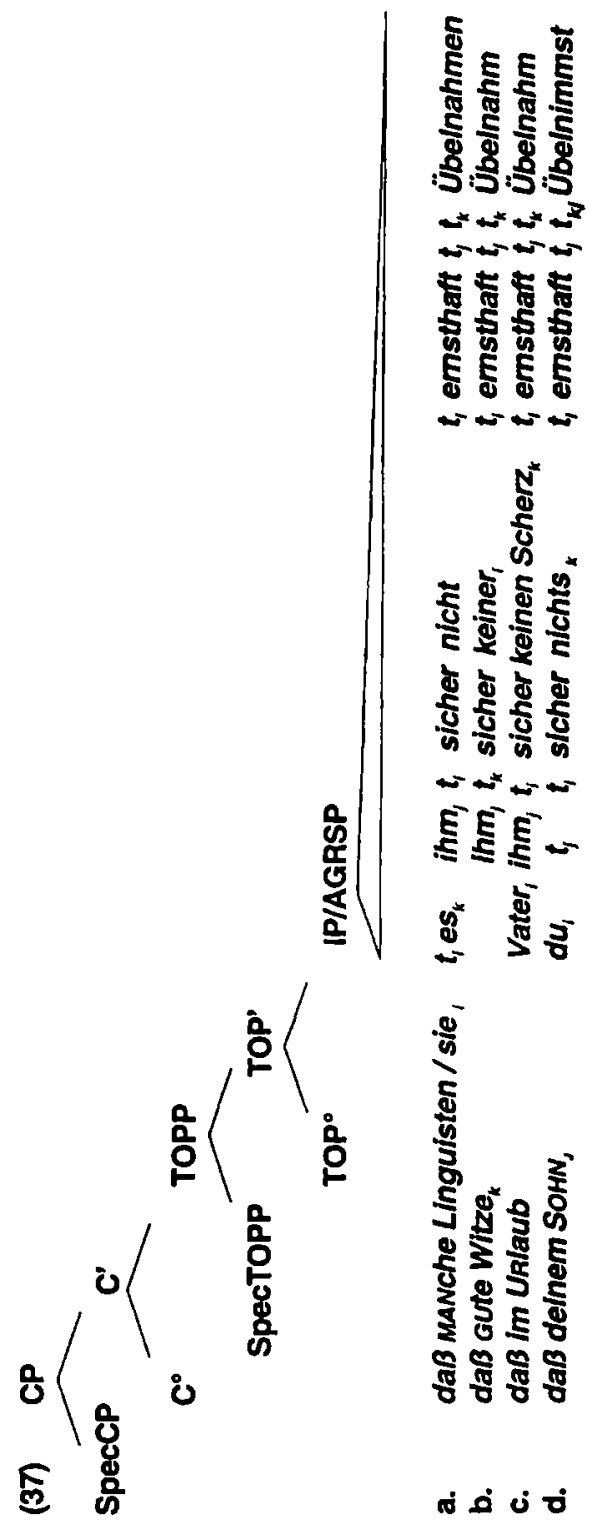


Das Topik ist immer dann sekundär betont, wenn es auf etwas referiert, das nicht unmittelbar vorerwähnt ist, vgl. in (37a): manche Linguisten mit Hutkontur vs, sie ohne diese Kontur.

Von der Topik-Position ausgeschlossen ist per definitionem stets die Phrase, die den Hauptakzent des Satzes trägt, also der Fokusexponent:

\section{*daß MUT Vater ihm zugesprochen hat}

Die Annahme, daß hier nur eine Position für eine Phrase zur Verfügung steht, entspricht dem empirischen Befund, daB vor den auf Vorerwähntes referierenden Phrasen normalerweise vom Hörer nur eine Phrase als Topik akzeptiert wird, s. (39-41):

(39) ? daß gestern manche Linguisten $t_{\mathrm{i}} e s_{\mathrm{k}}$ ihm $m_{\mathrm{j}} t_{\mathrm{i}}$ sicher nicht $t_{\mathrm{i}}$ ernsthaft $t_{\mathrm{j}} t_{\mathrm{k}}$ übelnahmen

(40) ? daß seitdem gute Witze $t_{k}$ ih $m_{j} t_{k}$ sicher keiner $t_{i}$ ernsthaft $t_{\mathrm{j}} t_{\mathrm{k}} \ddot{u}$ belnahm

(41) ? daß im Urlaub Vater $t_{\mathrm{i}}$ ih $m_{\mathrm{j}} t_{\mathrm{i}}$ sicher keinen Scher $z_{\mathrm{k}} t_{\mathrm{i}}$ ernsthaft $t_{\mathrm{j}} t_{\mathrm{k}}$ übelnahm

In deklarativen Verbzweit-Sätzen wandert nun im möglicherweise topikprominenten Deutschen die als Topik markierte Phrase in die als Vorfeld bekannte Position vor das finite Verb, d.h. in die Spezifiziererposition von CP, vgl. (42-44), es sei denn diese Position ist durch die den Hauptakzent des Satzes tragende maximale Phrase, die nie Topik sein kann, besetzt wie in (45):

(42) Viele Linguisten $n_{\mathrm{i}} /$ sie $_{\mathrm{i}}$ nahmen $t_{\mathrm{i}}$ es ${ }_{\mathrm{k}}$ ihm $\mathrm{m}_{\mathrm{j}} t_{\mathrm{i}}$ sicher nicht $t_{\mathrm{i}}$ ernsthaft $t_{\mathrm{j}} t_{\mathrm{k}}$ Übel

(43) Gute Witze nahm ihm $_{\mathrm{j}} t_{\mathrm{k}}$ sicher KEIner $t_{\mathrm{i}}$ ernsthaft $t_{\mathrm{j}} t_{\mathrm{k}}$ übel

(44) Im Urlaub nahm Vater, ihm $t_{\mathrm{j}} t_{\mathrm{i}}$ sicher KEInen Scher $z_{\mathrm{k}} t_{\mathrm{i}}$ ernsthaft $t_{\mathrm{j}} t_{\mathrm{k}}$ übel

(45) MUT hat Vater ihm zugesprochen

Ist weder die den Hauptakzent des Satzes tragende maximale Phrase in SpecCP angeordnet, noch eine Topikphrase vorhanden, dann wird häufig wie in (46) ein expletives $E S$ eingesetzt: ${ }^{11}$

(46) Es nahmen [AGROPihm $m_{\mathrm{j}}$ sicher [vP viele Linguisten $t_{\mathrm{j}}$ so manche Äußerung übel]]

11 Daneben gibt es (sicher häufiger in der gesprochenen Sprache) auch oft als narrativ bezeichnete deklarative V/1-Sătze vom Typ „Kam ein Hase zum Apotheker...” , „Hat (doch) gestern Anton Fritz getroffen und ...” usw. 
$\mathrm{Da} B$ dieses expletive Element für ein leeres Topik steht, beweist die geringere Grammatikalität von Sätzen wie (47)-(49), wo ein vorhandenes Topik nicht in die SpecCP-Position, also ins Vorfeld, verschoben worden ist:

(47) ? Es nahmen viele Linguisten $t_{\mathrm{i}} i h m_{\mathrm{j}}$ den Scher $z_{\mathrm{k}} t_{\mathrm{i}}$ sicher $t_{\mathrm{i}}$ ernsthaft $t_{\mathrm{j}} t_{\mathrm{k}}$ übel $t_{\mathrm{v}}$

(48) ? Es nahm gute Witze $t_{\mathrm{k}} t_{\mathrm{k}}$ ihm $m_{\mathrm{j}}$ sicher keiner $t_{\mathrm{i}}$ ernsthaft $t_{\mathrm{j}} t_{\mathrm{k}}$ übel $t_{\mathrm{v}}$

(49) ?? Es nahm im Urlaub Vater ${ }_{1} m_{\mathrm{j}} t_{\mathrm{i}}$ sicher keinen Scherz $\mathrm{z}_{\mathrm{k}} t_{\mathrm{i}}$ ernsthaft $t_{\mathrm{j}} t_{\mathrm{k}}$ übel $t_{\mathrm{v}}$

und die Ungrammatikalität von (50), wo das prototypische Topik, nämlich das pronominale Subjekt, nicht nach SpecCP ins Vorfeld gewandert ist:

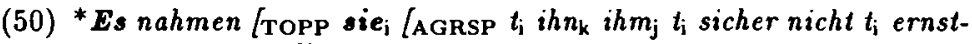
haft $t_{\mathrm{j}} t_{\mathrm{k}}$ übel $\left.t_{\mathrm{v}}\right]$

Im Deutschen können also durchaus viele statistisch mögliche Reihenfolgen von Subjekt- und Objektkonstituenten auch tatsächlich vorkommen, deren Anordnung unterliegt aber trotzdem strengen Restriktionen, ist also keineswegs frei im Sinne von beliebig. Deshalb komme ich zu dem meinen Titel einschränkenden SchluB: Deutsch ist eine abgeleitete V/2Sprache mit zugrundeliegender SOV-Reihenfolge. Die scheinbare Freiheit der Wortstellung ist streng restringiert.

\section{Literatur}

Abraham, W. (1992): Structural Properties of Information Packaging in German and in Universal Grammar. Erscheint in: Kiss, K.E. (ed.): Focus Languages. Oxford.

Abraham, W. (1995): Deutsche Syntax im Sprachenvergleich. Grundlegung einer typologischen Syntax des Deutschen. Tübingen. (= Studien zur deutschen Grammatik 41).

Brandt, M./Reis, M./Rosengren, I./Zimmermann, I. (BRRZ ) (1992): Satz, Satztyp und Illokution. In: Rosengren, Inger ( $\mathrm{Hg}_{\text {. }}$ ): Satz und Illokution. Bd.1. Tübingen. S. 1-90. (= Linguistische Arbeiten 278).

Bierwisch, M. (1990): Verb Cluster Formation as a Morphological Process. In: Yearbook of morphology 3, S. 173-199.

Chomsky, N. (1986): Barriers. Cambridge/Mass. MIT Press.

Chomsky, N. (1992): A Minimalist Program for Linguistic Theory. MIT Occasional Papers in Linguistics 1. Cambridge/Mass.

Donati, C./Tomaselli, A. (1995): Language Types and Generative Grammar: A Review of Some Consequences of the Universal VO Hypothesis. Ms. 
Haftka, B. (1981, 1984): Reihenfolgebeziehungen im Satz (Topologie). Kap. 4. In: Heidolph, K. E./Flämig, W./Motsch, W. (Hg.), S. 702-764.

Haftka, B. (1988a): Linksverschiebungen. Ein Beitrag zur Konfigurationalitāt des Deutschen. In: Bierwisch, M./Motsch, W./Zimmermann, I. (Hg.) (1988): Syntax, Semantik und Lexikon. Berlin. S. 89-146. (= studia grammatica XXIX).

Haftka, B. (1988b): Ob vielleicht tatsächlich nicht gern reist? In: Lang, E. (Hg.) (1988): Studien zum Satzmodus I. Berlin. S. 25-58. (= Linguistische Studien des ZISW der AdW der DDR R. A, 177).

Haftka, B. (1993): Topologische Felder und Versetzungsphänomene. In: Syntax. Ein internationales Handbuch zeitgenōssischer Forschung. Berlin/New York. S. 846-867.

Haftka, B. (Hg.) (1994): Was determiniert Wortstellungsvariation? Opladen.

Haftka, B. (1994): Wie positioniere ich meine Position? In: Haftka, B. (Hg.) (1994), S. 139-160.

Haftka, B. (1995): Syntactic Positions for Topic and Contrastive Focus in the German Middlefield. Vortrag bei der AG Fokus-Hintergrund-Gliederung bei der DGfS-Jahrestagung in Gōttingen. 1.-3. Mãrz 1995.

Hawkins, J. A. (1990): Word order universals. New York.

Heidolph, K.E./Flāmig, W./Motsch, W. (Hg.) (1981, 1984): Grundzüge einer deutschen Grammatik. Berlin.

Kayne, R. (1994): The Antisymmetry of Syntax. MIT Press.

Larson, R. (1988): On the Double Object Construction. In: Linguistic Inquiry 19, S. 335-391.

Molnár, V. (1991): Das TOPIC im Deutschen und im Ungarischen. Stockholm. (= Lunder Germanistische Forschungen 58).

Rosengren, I. (1994): Scrambling - was ist das? In: Haftka, (Hg.) (1994), S. 175-196.

Schmidt, C. M.(1995): Satzstruktur und Verbbewegung. Eine minimalistische Analyse zur internen Syntax der IP (INFLection-Phrase) im Deutschen. Tübingen. (= Linguistische Arbeiten 327).

Wegener, H. (1985): Der Dativ im heutigen Deutsch. Tübingen. (= Studien zur deutschen Grammatik 28).

Zimmermann, I. (1991): Die Syntax der Substantivgruppe. Weiterentwicklung der X'-Theorie. In: Zimmermann, I. (Hg.) (1991): Syntax und Semantik der Substantivgruppe. Berlin. S. 1-32. (= Studia grammatica XXXIII). 